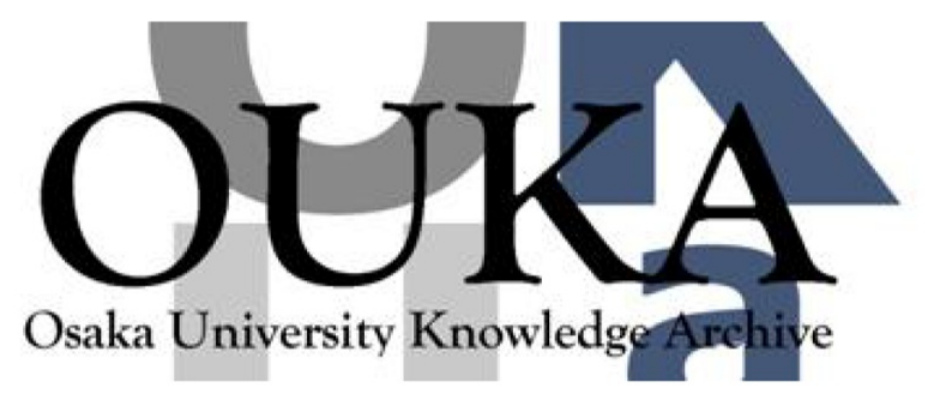

\begin{tabular}{|c|c|}
\hline Title & $\begin{array}{l}\text { Temperature Tuning of Optical Stop Band of } \\
\text { Liquid-Crystal Infiltrated Synthetic Opal }\end{array}$ \\
\hline Author (s) & $\begin{array}{l}\text { Shimoda, Yuki; Nakayama, Keizo; Ozaki, Masanori } \\
\text { et al. }\end{array}$ \\
\hline Citation & 電気材料技術雑誌. 9(2) p.5-p.6 \\
\hline Issue Date & $2000-04-06$ \\
\hline oaire:version & VoR \\
\hline URL & https://hdl. handle. net/11094/81584 \\
\hline rights & \\
\hline Note & \\
\hline
\end{tabular}

Osaka University Knowledge Archive : OUKA

https://ir. Library. osaka-u. ac. jp/

Osaka University 


\title{
Temperature Tuning of Optical Stop Band of Liquid-Crystal Infiltrated Synthetic Opal
}

\author{
Yuki Shimoda, Keizo Nakayama, Masanori Ozaki, Katsumi Yoshino \\ Department of Electronic Engineering, Graduate School of Engineering, Osaka University, \\ 2-1 Yamada-oka, Suita, Osaka 565-0871, Japan \\ Tel: +81-6-6879-7759, Fax: +81-6-6879-7774 \\ E-mail: yshimoda@ele.eng.osaka-u.ac.jp
}

Photonic crystals with a three-dimensional ordered structure with a periodicity of optical wavelength, have attracted much attention from both fundamental and practical points of view, because novel physical concepts such as photonic band gap have been theoretically and understand fundamental optical properties such as the stop band of the three-dimensional periodic structure.

Synthetic opals have been studied as pseudo photonic crystals or prototype photonic crystals in order to establish growth techniques.

In this study, we demonstrate that liquid crystals can be infiltrated into a three-dimensional periodic array of interconnected nanosize voids in synthetic opals and the the stop band can be tuned in synthetic opals infiltrated with liquid ctystals by controlling the refractive index predicted and various applications of photonic crystals have been proposed.

Ordered colloidal crystal in a film was formed in sandwich cells made of two glass plates with separation of 25 and $50 \mu \mathrm{m}$ by sedimentation of the suspension of monodispersed silica spheres of 300 and $1000 \mathrm{~nm}$ diameters. In this paper, the opals made of $300 \mathrm{~nm}$ and $1000 \mathrm{~nm} \mathrm{SiO} 2$ spheres and named as opal-300 and opal-1000, respectively. Figures 1 (a) and $1(\mathrm{~b})$ respectively show transmission spectra of the opal-300 and opal-1000 films for nomal incidence. As is evident in this figure, clear stop bands appear at the wavelengths of around 650 and $2250 \mathrm{~nm}$ in the opal-300 and opal-1000 were respectively. The diameters of $\mathrm{SiO}_{2}$ spheres of the opal-300 and opal-1000, evaluated from the observed diffraction and transmission spectra by assuming a fcc lattice, which coincide nearly with those of the original spheres.

Figure 2 indicates transmission spectra of the opal-300 and the opal-300 infiltrated with nematic liquid crystal (ZLI1132). As is evident in this figure, the stop band of the nematic liquid crystal infiltrated opal appears at longer wavelength by about $70 \mathrm{~nm}$ in comparison with that of the opal-300 itself, which should originate from the large refractive index of ZLI1132. It should also be noted in the inset of Fig. 2 that even in the same nematic liquid crystal infiltrated opal, the stop band shifts with changing temperature. From these spectral change, we can evaluate the refractive index of the liquid crystal by utilizing the periodicity of the opal and the refractive index of $\mathrm{SiO}_{2}$ as a function of temperature. Figure 3 shows the temperature dependence of the refrative index of ZLI1132. With decreasing temperature, stepwise increase of the refractive index was observed at the phase transition point between the isotropic and nematic phases.
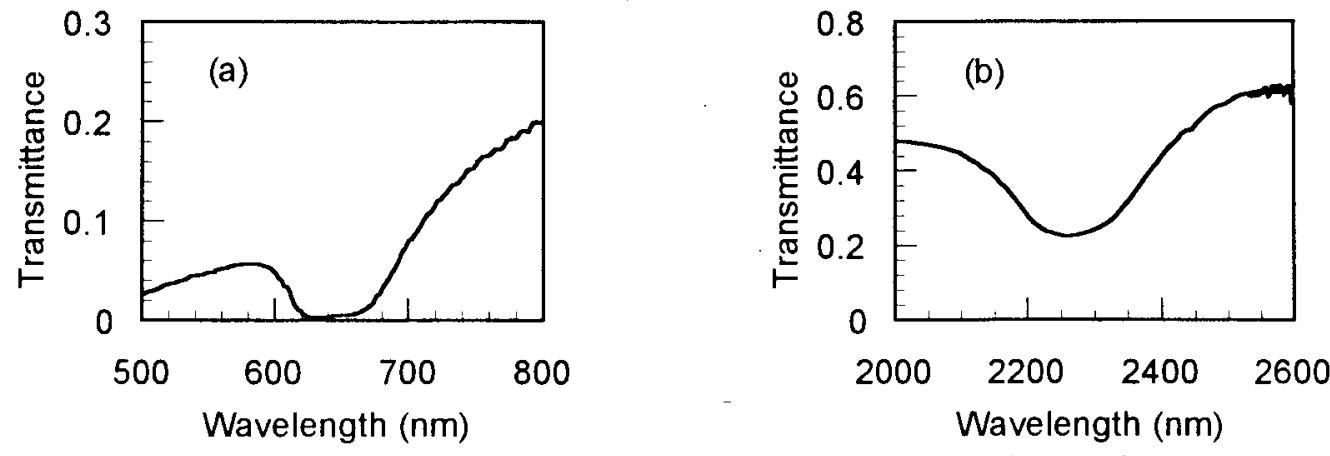

Fig 1. Transmission spectra of opal-300 (a) and opal-1000 (b) 


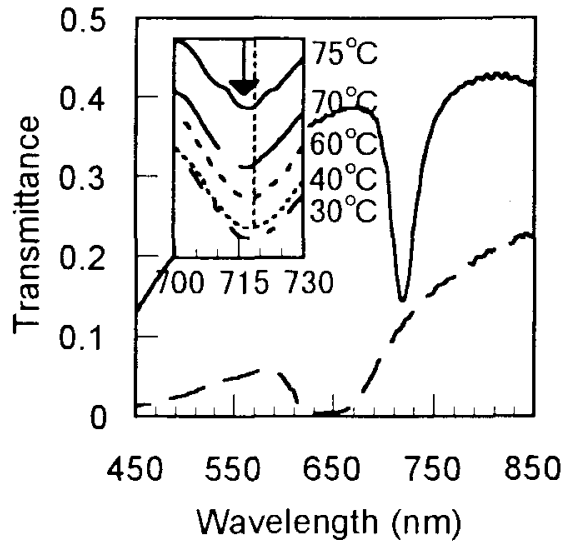

Fig.2. Transmission spectra of opal-300 (broken line)and opal-300 infiltrated with nematic liquid crystal (solid line). The inset shows the temperature dependence of transmission spectra.

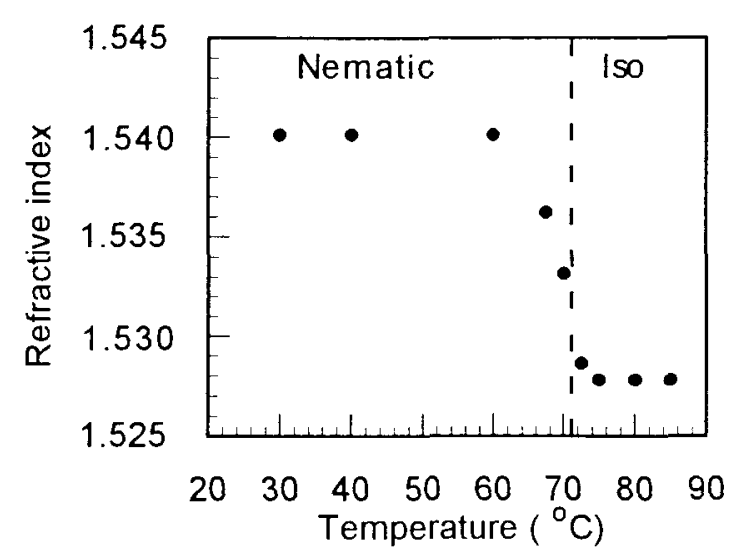

Fig.3. Temperature dependence of evaluated refractive index of nematic liquid crystal (ZLI1132) in voids of opal-300.

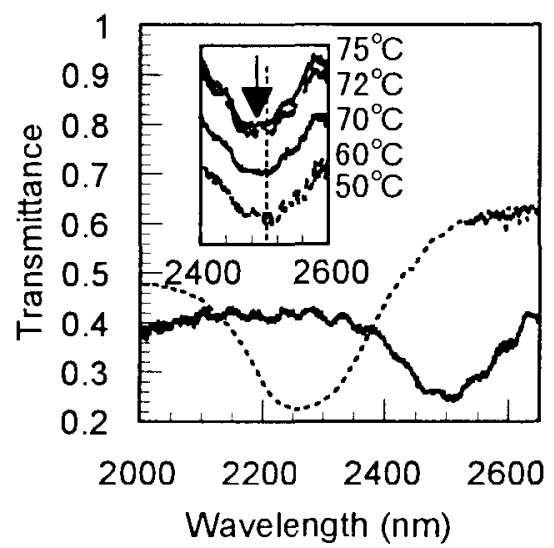

Fig.4. Transmission spectra of opal-1000 (broken line) and opal-1000 infiltrated with nematic liquid crystal (solid line). The inset shows the temperature dependence of transmission spectra.

The stop band of the opal-1000 infiltrated with nematic liquid crystal ZLI1 132 also exhibited remakable shift as shown in Fig.4. It should also be noted in the inset of this figure that the stop band of ZLI1132 infiltrated opal-1000 shifts with depending on the liquid crystal phases.

From the temperature dependence of the stop band wavelength, the refractive index of ZLI1132 in the opal-1000 can be evaluated. This result shows the stepwise change of the refractive index at the phase transition point.

In summary, we demonstrated that the stop band of the opal can be clearly tuned by infiltrating liquid crystals and changing the temperature, which seems to support the possibility of the tunable photonic crystals. It should also be mentioned that we demonstrated a measurement method for the refractive index. That is, by observing the transmission spectra through infiltrated opals, the refractive index of the infiltrated material can be evaluated.

\section{References}

This work was supported by NEDO International Joint Research Grant

[1] K.Yoshino. Y.Shimoda. Y.Kawagishi, K.Nakayama and M.Ozaki. Appl.Phys.Lett., 75. 932 (1999)

[2] S.John, Phys.Let1., 58. 2486 (1987)

[3] S.John and T.Quang. Phys.Rer.Lett. 74. 3419(1995)

[4] K.Yoshino. K. Tada. M.Ozaki. A. A.Zakihidov and R.H.Baughman. Jpn. J.Appl.Phys.. Part 2. 36. L714(1997)

[5] K.Yoshine. S.Tatsuhara. Y.Kawagishi. M.Ozaki. A. A.Zakhidov and Z.V.Vardeny. Appl.Phys.Lett. 74. 2590) (1999)

[6] K. Yoshino. Production Techinique 50.26 (1998) (in Japanease) 